\title{
Extrahepatic Manifestations of Nonalcoholic Fatty Liver Disease
}

\author{
Andrew A. Li ${ }^{1}$, Aijaz Ahmed², and Donghee Kim² \\ ${ }^{I}$ Department of Medicine and ${ }^{2}$ Division of Gastroenterology and Hepatology, Stanford University School of Medicine, Stanford, CA, USA
}

Nonalcoholic fatty liver disease (NAFLD) is the most common chronic liver disease and encompasses a spectrum of pathology from simple steatosis to inflammation and significant fibrosis that leads to cirrhosis. NAFLD and its comorbid conditions extend well beyond the liver. It is a multisystemic clinical disease entity with extrahepatic manifestations such as cardiovascular disease, type 2 diabetes, chronic kidney disease, hypothyroidism, polycystic ovarian syndrome, and psoriasis. Indeed, the most common causes of mortality in subjects with NAFLD are cardiovascular disease, followed by malignancies and then liver-related complications as a distant third. This review focuses on several of the key extrahepatic manifestations of NAFLD and areas for future investigation. Clinicians should learn to screen and initiate treatment for these extrahepatic manifestations in a prompt and timely fashion before they progress to end-organ damage. (Gut Liver 2020;14:168-178)

Key Words: Nonalcoholic steatohepatitis; Cardiovascular disease; Metabolic syndrome

\section{INTRODUCTION}

Nonalcoholic fatty liver disease (NAFLD) is defined by the presence of hepatic steatosis in the absence of other causes for hepatic fat accumulation, most commonly significant alcohol use, medications and other causes of chronic liver disease. NAFLD encompasses a spectrum of liver disease ranging from isolated hepatic steatosis characterized by intrahepatic triglyceride accumulation (nonalcoholic fatty liver, NAFL), steatosis with inflammation and hepatocyte injury (nonalcoholic steatohepatitis, NASH), NASH with fibrosis that can progress to end-stage liver disease (NASH-related cirrhosis), and potentially hepatocellular carcinoma. NAFLD has become increasingly common, with estimates of prevalence in the United States ranging from
$10 \%$ to $46 \% \%^{1-3}$ and worldwide up to $25 \%{ }^{1,4}$ Its rise in prevalence has closely paralleled with metabolic syndrome and individual components of metabolic syndrome such as central obesity, dyslipidemia, and type 2 diabetes mellitus (T2DM). ${ }^{5}$ Although NAFLD is sometimes perceived as the hepatic manifestation of the metabolic syndrome, there is now a growing body of evidence that NAFLD may, in fact, be a key driver in metabolic syndrome. The hepatic involvement is just one component of a multi-organ manifestation of NAFLD, with effects on the cardiovascular, renal, and endocrine systems, as well as the risk of extrahepatic malignancies. In fact, the leading cause of mortality in patients with NAFLD is cardiovascular disease, followed by extrahepatic malignancies, and then liver-related mortality. ${ }^{6,7}$ Therefore, physicians and patients should be aware of the multisystemic involvement of NAFLD without a clear pattern or order of clinical presentation. Therefore, a high level of clinical suspicion based on a patient risk profile is the most prudent approach. Widespread screening for NAFLD is not recommended at this time. This review will focus on the association between NAFLD and metabolic syndrome and the extrahepatic manifestations of NAFLD (Table 1).

\section{METABOLIC SYNDROME}

The metabolic syndrome is typically defined by the presence of at least three of following risk factors: central obesity, high blood pressure, high blood sugar, high serum triglycerides, and low serum high-density lipoprotein cholesterol. The metabolic syndrome is highly prevalent in subjects with NAFLD. The prevalence of metabolic syndrome increased with increasing body mass index (BMI), from 18\% in nonobese NAFLD to 67\% in obese-NAFLD in 304 subjects with NAFLD. ${ }^{8}$ Eighty-eight percent of subjects with NASH had metabolic syndrome (vs 53\% of subjects with NAFL). ${ }^{8}$ The presence of metabolic syndrome carried a high risk of NASH and severe fibrosis among subjects

Correspondence to: Donghee Kim

Division of Gastroenterology and Hepatology, Stanford University School of Medicine, 300 Pasteur Drive, Stanford, CA 94304, USA

Tel: +1-650-497-9261, Fax: +1-650-498-5692, E-mail: dhkimmd@stanford.edu

Received on February 20, 2019. Revised on March 26, 2019. Accepted on April 5, 2019. Published online June $17,2019$. pISSN 1976-2283 eISSN 2005-1212 https://doi.org/10.5009/gnl19069

(a) This is an Open Access article distributed under the terms of the Creative Commons Attribution Non-Commercial License (http://creativecommons.org/licenses/by-nc/4.0) which permits unrestricted non-commercial use, distribution, and reproduction in any medium, provided the original work is properly cited. 
Table 1. Key Extrahepatic Manifestations of NAFLD

\begin{tabular}{ll}
\hline \multicolumn{1}{c}{ Extrahepatic manifestation } & \multicolumn{1}{c}{ Key finding } \\
\hline Metabolic syndrome & Increasing prevalence of metabolic syndrome with progression of NAFLD, NASH, and severe fibrosis (18\%-88\%) \\
& Presence of metabolic syndrome associated with higher overall mortality in NAFLD \\
Visceral adiposity & Visceral adiposity carries a higher risk than subcutaneous adiposity for NAFLD \\
Type 2 diabetes & Insulin resistance is a common pathogenic mechanism for both type 2 diabetes and NAFLD, and more "severe" \\
& NAFLD is more likely to have incident diabetes \\
& Presence of type 2 diabetes in NAFLD increases mortality by 2.2-fold, and NAFLD increases the risk of micro- \\
& vascular diabetic complications \\
Cardiovascular disease & cular disease events and subclinical markers \\
& More "severe" forms of NAFLD associated with higher risk of cardiovascular disease events and mortality \\
Chronic kidney disease & More "severe" NAFLD increases the likelihood of renal impairment, and improvement in hepatic disease may \\
Hypothyroidism & also improve renal function \\
Psoriasis & Subclinical and overt hypothyroidism link with NAFLD \\
Polycystic ovarian syndrome & High prevalence of concurrent NAFLD and NASH in psoriasis \\
\hline
\end{tabular}

NAFLD, nonalcoholic fatty liver disease; NASH, nonalcoholic steatohepatitis.

with NAFLD after correction for sex, age, and BMI. ${ }^{8}$ A recent study from the NASH Clinical Research Network reported that metabolic syndrome had a 40\% increased risk of histologyconfirmed NASH. ${ }^{9}$ In an analysis using the National Health and Nutrition Examination and Survey (NHANES) III, the metabolic syndrome was independently associated with increased risk of overall mortality among subjects with NAFLD, although obesity was not associated with an increased risk of all-cause mortality in subjects with NAFLD. ${ }^{10}$

There may be a bidirectional relationship as well, with a study of approximately 1,000 participants of the Framingham Heart Study identifying that those with NAFLD at baseline were at higher risk to develop subsequent hypertension and T2DM than those without NAFLD, and those with elements of the metabolic syndrome were more likely to develop incident NAFLD. ${ }^{11}$ Kwon et al. ${ }^{12}$ showed that NAFLD was associated with a risk of having components of metabolic syndrome, and the association was stronger for nonobese NAFLD than for obese NAFLD. A metaanalysis reported that NAFLD, as diagnosed by either liver enzymes or ultrasonography, significantly increased the risk of incident metabolic syndrome during a 5-year follow-up period. ${ }^{13}$ In a pooled population of 81,411 , NAFLD was associated with increased risk of incident metabolic syndrome with a relative risk of 1.80 for alanine aminotransferase (last vs first quartile or quintile), 1.98 for gamma-glutamyltransferase, and 3.22 for ultrasonography. ${ }^{13}$

\section{VISCERAL ADIPOSITY}

The prevalence of NAFL on biopsy in patients undergoing bariatric surgery for morbid obesity ranges up to 90\%; NASH was seen in $30 \%$ to $50 \%$, and up to $5 \%$ had cirrhosis. ${ }^{14-17}$ In addition, visceral adiposity appears to confer a higher risk for NAFLD compared to subcutaneous fat deposition. A longitudinal study of approximately 2,000 subjects identified that larger areas of visceral adipose tissue (VAT) at baseline was associated with higher incident NAFLD, with a hazard ratio (HR) of 2.23 in the highest quintile after adjusting for other factors, whereas an association with subcutaneous adipose tissue was nonexistent. ${ }^{18}$ In contrast, higher areas of subcutaneous adipose tissue were longitudinally associated with regression of NAFLD. ${ }^{18}$ Increases in VAT area over time also correlate higher likelihood of incident NAFLD, while a decrease in VAT over time was correlated with the likelihood of regressed NAFLD. ${ }^{19}$ Within NAFLD, VAT area also predicts the likelihood of NAFL, NASH, and NAFLD with fibrosis, with higher areas of VAT associated with more advanced liver disease. ${ }^{20}$ In summary, these data indicate that certain types of body fat are risk factors for NAFLD, whereas other types could reduce the risk for NAFLD. Visceral obesity is most likely an important target for future interventions in the treatment of NAFLD and advanced fibrosis.

\section{TYPE 2 DIABETES}

NAFLD and T2DM share common pathogenic pathways including obesity and insulin resistance. NAFLD is insulin resistant and therefore does not adequately suppress hepatic glucose production, and patients with both T2DM and NAFLD often have poor glycemic control, as compared to those with only T2DM without NAFLD. ${ }^{21,22}$ A recent meta-analysis in a pooled population of 296,439 subjects determined that NAFLD significantly increased the risk of incident T2DM with a pooled HR 
of 2.22 (95\% confidence intervals [CI], 1.84 to 2.60$).{ }^{23}$ Subjects with more "severe" NAFLD were also more likely to develop incident diabetes. ${ }^{23}$ However, there are likely complex bidirectional links between the two diseases. Approximately 75\% of subjects with T2DM have concurrent NAFLD, and the diagnosis of NAFLD in subjects with T2DM increases the risk of all-cause mortality by 2.2 -fold. ${ }^{24}$ Microvascular diabetic complications are also seen at higher rates in T2DM patients with concurrent NAFLD as compared to those without, with higher rates of both diabetic nephropathy and retinopathy. ${ }^{25-27} \mathrm{~T} 2 \mathrm{DM}$ also appears to exert effects on the progression of NAFLD, with incident T2DM being the most predictive factor for progression of NAFLD to NASH and advanced fibrosis. ${ }^{28}$

\section{CARDIOVASCULAR DISEASE}

Cardiovascular disease (CVD) is the leading cause of mortality in patients with NAFLD. Classical CVD risk factors such as hypertension, dyslipidemia, insulin resistance, smoking, and central obesity, share a strong overlap with both the metabolic syndrome and risk factors for NAFLD. ${ }^{8}$ These shared risk factors, many encapsulated by the metabolic syndrome, intimately link CVD and NAFLD, but there is growing evidence that the presence of NAFLD confers additional risk of premature CVD. This has potentially important clinical implications for risk factor reduction and screening. Additional shared risk factors between NAFLD and CVD are also emerging, along with altered levels of interleukin 6, adiponectin, tumor necrosis factor alpha, vitamin $\mathrm{D}$, fibrinogen, plasminogen, vascular adhesion molecules, and C-reactive protein, with many of these being liver-synthesized proteins (Table 2). ${ }^{25,29-51}$

A large body of evidence links NAFLD with atherosclerotic plaque formation and subclinical markers of CVD. ${ }^{52}$ Several cross-sectional studies linked NAFLD with increased carotid in- tima-media thickness, a well-validated tool for assessing atherosclerosis in asymptomatic patients that independently predicts CVD events. ${ }^{53-55}$ A meta-analysis including approximately 3,500 subjects also reported that NAFLD based on ultrasonography is significantly associated with carotid intima-media thickness and carotid plaques. ${ }^{56}$ Presence of NAFLD is also independently associated in a dose-dependent manner with higher cardio-ankle vascular indices, a score which represents the stiffness of whole arterial segments from the aorta to the ankle that is closely associated with coronary atherosclerosis, cardiac function, hypertension, stroke. ${ }^{57}$ Other case-control studies have also demonstrated an association of NAFLD with increase arterial wall stiffness, ${ }^{58,59}$ altered endothelium-dependent flow-mediated vasodilation. ${ }^{29,60}$ Large cross-sectional studies have also established the association between ultrasonography-defined NAFLD and coronary artery calcification independent of classical coronary risk factors. ${ }^{61-63}$ These three studies include more than 20,000 subjects and used computed tomography-based coronary artery calcium scores $^{61,63}$ or coronary angiography ${ }^{64}$ to determine coronary artery calcification. In a longitudinal study, NAFLD has also been shown to play an important role in the initial development of coronary artery calcification without any at baseline calcification. ${ }^{65}$

In terms of CVD events, epidemiological associations point to an association between NAFLD and the risk of cardiovascular events. In a study of 17,350 subjects without known liver disease or significant alcohol consumption, ultrasonographicallydetected NAFLD was associated with an elevated 10-year risk of CVD as estimated using the Framingham risk score (FRS), independent of classical risk factors and other components of the metabolic syndrome. ${ }^{66}$ NAFLD had an odds ratio (OR) of 1.35 (95\% CI, 1.10 to 1.65) of higher 10-year risk of CVD with FRS $>20 \%$ in a multivariate model after for controlling for age, gender, BMI, waist circumference, and individual components

Table 2. Pathophysiologic Mechanism Linking NAFLD and Cardiovascular Disease

\begin{tabular}{lc}
\multicolumn{1}{c}{ Pathophysiologic mechanism } & References \\
\hline Insulin resistance and type 2 diabetes & $21,29-31$ \\
Obesity & $14,15,17,20,21,32-34$ \\
Hypertension & 5,8 \\
Dyslipidemia & $5,8,35$ \\
Increased: LDL, triglycerides, VLDL & $36-41$ \\
Decreased: HDL & $36,42-45$ \\
Proinflammatory mediators & 46,47 \\
Increased: C-reactive protein, interleukin-6, tumor necrosis factor $\alpha$, reactive oxygen species & \\
Decreased: adiponectin &
\end{tabular}

NAFLD, nonalcoholic fatty liver disease; LDL, low-density lipoprotein cholesterol; VLDL, very-low-density lipoprotein cholesterol; HDL, highdensity lipoprotein cholesterol. 
of the metabolic syndrome. A recent meta-analysis including a pooled population of 34,043 adults showed that NAFLD significantly increased the risk of fatal and/or non-fatal CVD events during a median 6.9 years follow-up period, with a random effect OR of 1.64 (95\% CI, 1.26 to 3.75). ${ }^{67}$ More severe NAFLD was also more likely to develop fatal and nonfatal CVD events (OR, 2.58; 95\% CI, 1.78 to 3.75). ${ }^{67}$ Regarding mortality, an analysis of the NHANES III in the United States, with a mean follow-up period of 15 years for NAFLD as defined by noninvasive scoring systems demonstrated increased mortality from CVD in subjects with advanced fibrosis. ${ }^{7}$ Indeed, another cohort study also supported the notion that more advanced fibrosis in NASH is associated with higher risk of CVD-related mortality and liverrelated disease. ${ }^{68}$ A multinational study of 458 patients with biopsy-confirmed NAFLD with bridging fibrosis or cirrhosis reported that patients with NAFLD cirrhosis have predominantly liver-related events, whereas those with bridging fibrosis have predominantly non-hepatic cancers and CVD events. ${ }^{69}$

Taken together, subjects with NAFLD are at high risk for CVD, including carotid and coronary atherosclerosis beyond what is explained by classical cardiovascular risk factors, visceral adiposity, and metabolic syndrome. Subjects with NAFLD should undergo careful cardiovascular surveillance. Moreover, those with the more severe forms of NAFLD need particular attention to ameliorate their high risk of CVD mortality.

\section{CHRONIC KIDNEY DISEASE}

Chronic kidney disease (CKD), defined as decreased estimated glomerular filtration rate less than $60 \mathrm{~mL} / \mathrm{min} / 1.73 \mathrm{~m}^{2}$, abnormal albuminuria or overt proteinuria, is observed at high rates in subjects with NAFLD diagnosed by imaging or biopsy, ranging from approximately 20\% to 50\% in those with NAFLD compared to $5 \%$ to $25 \%$ in those without, ${ }^{25-27,70-79}$ which in most studies was independent of common risk factors for CKD such as hypertension, T2DM, and other renal risk factors. A metaanalysis totaling nearly 64,000 subjects demonstrated that NAFLD, as diagnosed by either noninvasive scoring systems, imaging, or histology, was associated with an approximately 2-fold increase in both prevalent (OR, 2.12; 95\% CI, 1.69 to 2.66) and incident CKD (HR, 1.79; 95\% CI, 1.65 to 1.95$){ }^{80}$ There additionally appears to be a greater degree of renal impairment with histological severity of NASH. ${ }^{26}$ Indeed, a meta-analysis mentioned earlier demonstrated that NASH was associated with a higher prevalence (OR, 2.53; 95\% CI, 1.58 to 4.05 ) and incidence (HR, 2.12; 95\% CI, 1.42 to 3.17) of CKD than NAFL. ${ }^{80}$ Additionally, advanced fibrosis was associated with a higher prevalence (OR, 5.20; 95\% CI, 3.14 to 8.61) and incidence (HR, 3.29; 95\% CI, 2.30 to 4.71 ) of CKD than non-advanced fibrosis. ${ }^{80}$ More intriguingly, there is evidence that in patients with biopsy-proven NASH being treated with lifestyle modification over a year, improvement in NASH and histological findings were indepen- dently associated with improvement in renal function. ${ }^{81}$ Emerging mechanistic links between NAFLD and CKD include altered regulation of angiotensin-converting enzyme 2, impaired antioxidant defense mediated by nuclear factor erythroid 2-related factor-2, lipoprotein dysmetabolism, altered intestinal barrier integrity, and microbiome disturbances. ${ }^{82}$ Although the mechanism of renal injury is postulated to be mediated primarily through atherogenesis, the absence of renal biopsies in studies examining NAFLD and CKD makes this an open question. These findings suggest that the strong association between NAFLD and $\mathrm{CKD}$, including a relationship that appears to correspond with the severity of NAFLD, warrants clinical consideration in that improvement of NAFLD may also improve CKD, and that management of CKD and its sequelae should be incorporated in the care of patients with NAFLD.

\section{HYPOTHYROIDISM}

The thyroid gland plays an integral part in maintaining metabolic homeostasis, with effects on obesity, dyslipidemia, and therefore may be linked with NAFLD. ${ }^{83}$ Chung et al. ${ }^{83}$ determined that subclinical hypothyroidism was related to NAFLD in a dose-dependent manner. A cross-sectional study of 2,324 cases with hypothyroidism with age- and sex-matched controls demonstrated a higher prevalence of NAFLD with both increased thyroid-stimulating hormone (TSH) and decreased free thyroxine (T4), including with subclinical hypothyroidism and overt hypothyroidism. ${ }^{83}$ Multivariate analysis showed that subclinical and overt hypothyroidism are closely associated with NAFLD independent of the metabolic risk factors. ${ }^{83} \mathrm{~A}$ study using biopsy-proven NAFLD cohorts identified a higher prevalence (21\% vs 9.5\%) of hypothyroidism versus controls matched for age, sex, ethnicity, BMI, and metabolic syndrome components. ${ }^{84}$ A cross-sectional study of 425 subjects with biopsy-proven NAFLD determined that the prevalence of NASH and advanced fibrosis were significantly higher in subjects with low thyroid function (TSH $\geq 2.5 \mathrm{mIU} / \mathrm{L}$ ) versus strict-normal (TSH, 0.4 to $2.5 \mathrm{mIU} / \mathrm{L})$ thyroid function (52.4\% vs $37.2 \%$ for $\mathrm{NASH}$, $21.0 \%$ vs $10.6 \%$ for advanced fibrosis, $\mathrm{p}<0.01) .{ }^{85}$ Multivariate analyses showed that "low thyroid function" was significantly associated with NASH (OR, 1.61; 95\% CI, 1.04 to 2.50) and advanced fibrosis (OR, 2.23; 95\% CI, 1.18 to 4.23 ). ${ }^{85}$ The effects of plasma TSH within the euthyroid range on histological damage associated with NAFLD, found that low-normal thyroid function (TSH, 2.5 to 4.5) may also produce negative health effects similar to overt and subclinical hypothyroidism. ${ }^{85}$ Subclinical hypothyroidism, even in the range of upper normal TSH levels, was correlated to NAFLD in a dose-dependent manner. ${ }^{85}$ This Asian study was confirmed by another study based on U.S. NHANES 2007 to 2012. The prevalence of advanced fibrosis was significantly higher in subjects with low-normal thyroid function and subclinical hypothyroidism than those with strict-normal thy- 
roid function. ${ }^{86}$ In this study, multivariate analysis showed that "low-normal" thyroid function and subclinical hypothyroidism were significantly associated with a 1.9-fold increase (OR, 1.94; 95\% CI, 1.10 to 3.44) and 2.1-fold increase (OR, 2.05; 95\% CI, 1.01 to 4.16 ) in the risk for advanced fibrosis, respectively ( $p$ for trend $=0.005){ }^{86}$ A recent study hypothesized that thyroid hormone receptor may activate hepatic stellate cells, suggesting the potential role of thyroid hormone signaling in hepatic fibrogenesis. ${ }^{87}$ Currently, an orally administered, small-molecule liver-directed thyroid hormone receptor b agonist (MGL-3196) is under development for the treatment of NASH and hyperlipidemia. ${ }^{88,89}$ This hypothesis remains an area of open investigation, and further studies are warranted to elucidate the exact role of thyroid dysfunction in the progression to NASH and related advanced fibrosis.

\section{POLYCYSTIC OVARIAN SYNDROME}

Polycystic ovarian syndrome (PCOS) is characterized by hyperandrogenism, polycystic appearing ovaries, and oligomenorrhea or amenorrhea and occurs in 5\% to $18 \%$ of women. ${ }^{90}$ The prevalence of NAFLD within the PCOS population is estimated to be $15 \%$ to $55 \%$ depending on the diagnostic criteria and population. ${ }^{91}$ Conversely, a small study of female patients at a liver clinic identified a prevalence of $71 \%$ for PCOS amongst reproductive-aged women with NAFLD, and those with PCOS had a high prevalence of NASH. ${ }^{92}$ Indeed, several meta-analyses have demonstrated that in women with PCOS, there is a higher risk of co-existing NAFLD compared to matched controls with estimates ranging from 2.2-fold to 3.9-fold, independent of features of the metabolic syndrome. ${ }^{93-95}$ Like NAFLD, PCOS is associated with T2DM and insulin resistance, with approximately $50 \%$ to $80 \%$ of women with PCOS exhibiting the latter. ${ }^{96}$ Insulin resistance may directly contribute to the pathogenesis of PCOS. Interestingly, multiple studies have now shown that women with PCOS and hepatic steatosis have increased levels of insulin resistance compared to women with PCOS without steatosis. ${ }^{97,98}$ In women with PCOS, elevated alanine aminotransferase levels were also associated with insulin resistance as measured by euglycemic hyperinsulinemic clamp measures whereas it is similar to healthy controls in those with normal alanine aminotransferase. $^{99}$ Similarly, approximately $60 \%$ of women with PCOS are also obese, and 50\% have metabolic syndrome. ${ }^{91}$ However, a recent study reported that women with PCOS had a higher prevalence of NAFLD than those without in nonobese population. ${ }^{100}$ Hyperandrogenism was a risk factor for nonobese NAFLD irrespective of age, obesity, lipid profile, insulin resistance or glycemic status, suggesting an independent contribution of hyperandrogenism to NAFLD in nonobese women with PCOS. ${ }^{100}$ What is clear from the data is that there is are several common shared risk factors for both PCOS and NAFLD. Thus, careful evaluation for comorbid NAFLD and PCOS is warranted, especially in light of data suggesting higher rates of NASH in this population. ${ }^{92}$

\section{PSORIASIS}

Psoriasis is a chronic inflammatory disease with an estimated prevalence of $2 \%$ to $3 \%{ }^{101}$ and has been observed to have a higher prevalence in subjects with coexisting metabolic and/or obesity. ${ }^{101-103}$ NAFLD is also observed at a higher prevalence in subjects with psoriasis. One study of 129 subjects with psoriasis or psoriatic arthritis found that NAFLD occurs in approximately $47 \%$ of subjects with psoriasis, and $22 \%$ of subjects with psoriasis also had biopsy-proven NASH. ${ }^{104}$ Other studies have similarly observed this association and moreover demonstrated an adjusted OR of 1.7 for ultrasonographically-identified NAFLD in elderly subjects (age $>55$ years) with psoriasis as compared to those without, independent of alcohol consumption, smoking status, and presence of the metabolic syndrome. ${ }^{105}$ Interestingly, NAFLD was also associated with severity of psoriasis independent of age, gender, BMI, duration of psoriasis, and alcohol consumption. ${ }^{106}$ Psoriasis-associated NAFLD was more likely to have higher estimated liver fibrosis based on noninvasive scoring systems. ${ }^{107}$ Whether psoriasis and NAFLD are caused by common underlying mechanisms, or if one affects the incidence of the other remains undefined. Notably, there exists evidence suggesting that patients with psoriasis, metabolic syndrome, and NAFLD treated with etanercept, a tumor necrosis factor $\alpha$ inhibitor, as compared to those treated with psoralen-ultraviolet A had reductions in aspartate transaminase/alanine transaminase ratio, C-reactive protein serum levels, increased insulin sensitivity. ${ }^{108}$ Prospective studies are still needed to determine the impact of biologic treatments on NAFLD in psoriasis. ${ }^{109}$

\section{TREATMENT}

Currently, in the absence of approved effective pharmacologic treatment for NAFLD, the treatment of choice for NAFLD is weight loss with lifestyle modification. The same treatment strategy may be applied for extrahepatic manifestations, with lifestyle modification being a key component of treatment strategy in the control of blood glucose, blood pressure, hyperlipidemia, cardiovascular disease, and other risk factors. ${ }^{110,111}$ In addition, clinicians should have a higher index of suspicion for common extrahepatic manifestations in patients with NAFLD. A proposed screening strategy for the more common extrahepatic has been proposed by VanWagner and Rinella, ${ }^{112}$ which includes monitoring hemoglobin A1c, fasting glucose, blood pressure, lipid profile, urine microalbumin and albumin/creatinine ratio, estimated glomerular filtration rate, thyroid function tests, and ovarian ultrasound and/or serum androgens.

\section{Weight loss}

According to the American Association for the Study of Liver 
Disease practice guideline, loss of at least 3\% to 5\% of body weight may improve NAFLD, but a greater weight loss of up to $10 \%$ may be necessary to improve the degree of hepatic necroinflammation. ${ }^{113}$ By the same token, sustained weight reduction by $5 \%$ to $7 \%$ may be sufficient to lower the risk of T2DM. ${ }^{114}$ Recent intervention trials have shown a remarkable reduction in the risk of T2DM (of 42\% to 67\%) with weight reduction compared with control groups, even when the weight reduction was overall modest. ${ }^{114,115}$ Weight loss is associated with improvement in cellular insulin signal transduction, peripheral insulin sensitivity, and insulin secretory responses. ${ }^{115}$ Patients with patatinlike phospholipase domain-containing protein 3 (PNPLA3) NAFLD appear to be more sensitive to the beneficial effects of lifestyle modification on hepatic steatosis. ${ }^{116}$ Two European studies showed that weight loss decreases intrahepatic triglyceride concentration ${ }^{117}$ and liver enzymes ${ }^{118}$ even more in subjects with homozygous GG than in those with homozygous CC. The pathophysiology of this finding is still unknown-the differences in insulin sensitivity between two alleles and the effect on abdominal obesity, which may modulate the effect of PNPLA3 $\mathrm{G}$ allele on liver damage, may provide the explanations. ${ }^{117,118}$

\section{Diet}

Several dietary strategies have potentially positive effects on NAFLD, metabolic syndrome, and CVD. Reduction in the total calorie consumption is a crucial aspect of lifestyle modification, though at this time there is no consensus recommendation for dietary interventions to treat NAFLD, metabolic syndrome, and CVD. Daily caloric intake varies according to ethnicity, sex, BMI, and comorbidities. To achieve the optimal caloric reduction, estimation of individual energy requirements and prescription of an energy deficit of $500 \mathrm{kcal} /$ day or $30 \%$ of baseline is generally recommended. ${ }^{119}$ In addition to the reduction of the total caloric intake, a change in the composition of the diet may be important in the treatment of NAFLD and comorbid metabolic syndrome and/or CVD. Subjects with NAFLD tend to consume more soft drinks and meat, and less fish rich in omega-3 fatty acids. ${ }^{120}$ Recent systematic reviews have reported that restriction of dietary carbohydrate (e.g., simple carbohydrate and high glycemic carbohydrate) and fat (e.g., total and saturated fat) can lower the liver enzymes and/or reduce the grade of steatosis in subjects with NAFLD. ${ }^{121}$ To the extent that high fructose is associated with NAFLD and advanced histology, ${ }^{122}$ limiting fructose consumption may be beneficial.

\section{Physical activity}

Increased physical activity is thought to have a beneficial effect on NAFLD and comorbid conditions including metabolic syndrome and CVD by reducing visceral fat. Several studies have suggested that a reduction in hepatic fat was secondary to a reduction in visceral fat. Thus, the relationship between hepatic fat content and physical activity disappears when accounting for intra-abdominal obesity, ${ }^{123-125}$ although conflicting data exist. ${ }^{126-128}$ In a large cross-sectional study, an inverse association between total and leisure-time physical activities and the prevalence of NAFLD was observed, independent of visceral adiposity and insulin resistance. ${ }^{129}$ A recent prospective cohort study from our group demonstrated a lower risk of incident NAFLD in 4 years of follow-up based on physical activity level at baseline. ${ }^{130}$ Furthermore, sustained or increased physical activity had a preventive effect on incident NAFLD, independent of visceral adiposity and insulin resistance. ${ }^{130}$ In summary, increased physical activity is an important component of lifestyle modification in patients with NAFLD and comorbid extrahepatic manifestations, irrespective of visceral obesity or insulin resistance. However, there is no consensus regarding the most effective exercise regimen, such as duration and type of activities.

\section{CONCLUSION}

Based on current evidence, the clinical burden of NAFLD extends well beyond liver-related morbidity and mortality. NAFLD can be associated with extrahepatic complications including CVD, CKD, T2DM, hypothyroidism, PCOS, psoriasis, and metabolic syndrome. Though the majority of evidence to date is observational and retrospective, these associations have important clinical significance in screening, risk factor modification, and potential therapeutics. For example, weight loss, smoking cessation, and dietary changes have the potential to affect the progression of NAFLD and its extrahepatic comorbid complications, but future studies will be needed to better understand the pathophysiology and to potentially alter the natural history of these conditions.

\section{CONFLICTS OF INTEREST}

No potential conflict of interest relevant to this article was reported.

\section{ORCID}

Andrew A. Li

https://orcid.org/0000-0002-1295-8115

Aijaz Ahmed

https://orcid.org/0000-0002-3609-8586

Donghee Kim

https://orcid.org/0000-0003-1919-6800

\section{REFERENCES}

1. Williams CD, Stengel J, Asike MI, et al. Prevalence of nonalcoholic fatty liver disease and nonalcoholic steatohepatitis among a largely middle-aged population utilizing ultrasound and liver biopsy: a prospective study. Gastroenterology 2011;140:124-131.

2. Lazo M, Hernaez R, Eberhardt MS, et al. Prevalence of nonalcoholic fatty liver disease in the United States: the Third National Health and Nutrition Examination Survey, 1988-1994. Am J Epi- 
demiol 2013;178:38-45.

3. Vernon G, Baranova A, Younossi ZM. Systematic review: the epidemiology and natural history of non-alcoholic fatty liver disease and non-alcoholic steatohepatitis in adults. Aliment Pharmacol Ther 2011;34:274-285.

4. Younossi ZM, Koenig AB, Abdelatif D, Fazel Y, Henry L, Wymer M. Global epidemiology of nonalcoholic fatty liver disease-metaanalytic assessment of prevalence, incidence, and outcomes. Hepatology 2016;64:73-84.

5. Kim D, Touros A, Kim WR. Nonalcoholic fatty liver disease and metabolic syndrome. Clin Liver Dis 2018;22:133-140.

6. Adams LA, Lymp JF, St Sauver J, et al. The natural history of nonalcoholic fatty liver disease: a population-based cohort study. Gastroenterology 2005;129:113-121.

7. Kim D, Kim WR, Kim HJ, Therneau TM. Association between noninvasive fibrosis markers and mortality among adults with nonalcoholic fatty liver disease in the United States. Hepatology 2013;57:1357-1365.

8. Marchesini G, Bugianesi E, Forlani G, et al. Nonalcoholic fatty liver, steatohepatitis, and the metabolic syndrome. Hepatology 2003;37:917-923.

9. Brunt EM, Kleiner DE, Wilson LA, Belt P, Neuschwander-Tetri BA; NASH Clinical Research Network (CRN). Nonalcoholic fatty liver disease (NAFLD) activity score and the histopathologic diagnosis in NAFLD: distinct clinicopathologic meanings. Hepatology 2011;53:810-820.

10. Stepanova M, Rafiq N, Younossi ZM. Components of metabolic syndrome are independent predictors of mortality in patients with chronic liver disease: a population-based study. Gut 2010; 59:1410-1415.

11. Ma J, Hwang SJ, Pedley A, et al. Bi-directional analysis between fatty liver and cardiovascular disease risk factors. J Hepatol 2017;66:390-397.

12. Kwon YM, Oh SW, Hwang SS, Lee C, Kwon H, Chung GE. Association of nonalcoholic fatty liver disease with components of metabolic syndrome according to body mass index in Korean adults. Am J Gastroenterol 2012;107:1852-1858.

13. Ballestri S, Zona S, Targher G, et al. Nonalcoholic fatty liver disease is associated with an almost twofold increased risk of incident type 2 diabetes and metabolic syndrome. Evidence from a systematic review and meta-analysis. J Gastroenterol Hepatol 2016;31:936-944.

14. Machado M, Marques-Vidal P, Cortez-Pinto H. Hepatic histology in obese patients undergoing bariatric surgery. J Hepatol 2006;45:600-606.

15. Frantzides CT, Carlson MA, Moore RE, et al. Effect of body mass index on nonalcoholic fatty liver disease in patients undergoing minimally invasive bariatric surgery. J Gastrointest Surg 2004;8:849-855.

16. Abrams GA, Kunde SS, Lazenby AJ, Clements RH. Portal fibrosis and hepatic steatosis in morbidly obese subjects: a spectrum of nonalcoholic fatty liver disease. Hepatology 2004;40:475-483.
17. Dallal RM, Mattar SG, Lord JL, et al. Results of laparoscopic gastric bypass in patients with cirrhosis. Obes Surg 2004;14:47-53.

18. Kim D, Chung GE, Kwak MS, et al. Body fat distribution and risk of incident and regressed nonalcoholic fatty liver disease. Clin Gastroenterol Hepatol 2016;14:132-138.

19. Kim D, Chung GE, Kwak MS, Kim YJ, Yoon JH. Effect of longitudinal changes of body fat on the incidence and regression of nonalcoholic fatty liver disease. Dig Liver Dis 2018;50:389-395.

20. Yu SJ, Kim W, Kim D, et al. Visceral obesity predicts significant fibrosis in patients with nonalcoholic fatty liver disease. Medicine (Baltimore) 2015;94:e2159.

21. Anstee QM, Targher G, Day CP. Progression of NAFLD to diabetes mellitus, cardiovascular disease or cirrhosis. Nat Rev Gastroenterol Hepatol 2013;10:330-344.

22. Targher G, Bertolini L, Padovani R, et al. Prevalence of nonalcoholic fatty liver disease and its association with cardiovascular disease among type 2 diabetic patients. Diabetes Care 2007;30:1212-1218.

23. Mantovani A, Byrne CD, Bonora E, Targher G. Nonalcoholic fatty liver disease and risk of incident type 2 diabetes: a meta-analysis. Diabetes Care 2018;41:372-382.

24. Adams LA, Harmsen S, St Sauver JL, et al. Nonalcoholic fatty liver disease increases risk of death among patients with diabetes: a community-based cohort study. Am J Gastroenterol 2010;105:1567-1573.

25. Targher G, Bertolini L, Chonchol M, et al. Non-alcoholic fatty liver disease is independently associated with an increased prevalence of chronic kidney disease and retinopathy in type 1 diabetic patients. Diabetologia 2010;53:1341-1348.

26. Targher G, Bertolini L, Rodella S, Lippi G, Zoppini G, Chonchol M. Relationship between kidney function and liver histology in subjects with nonalcoholic steatohepatitis. Clin J Am Soc Nephrol 2010;5:2166-2171.

27. Targher G, Bertolini L, Rodella S, et al. Non-alcoholic fatty liver disease is independently associated with an increased prevalence of chronic kidney disease and proliferative/laser-treated retinopathy in type 2 diabetic patients. Diabetologia 2008;51:444-450.

28. McPherson S, Hardy T, Henderson E, Burt AD, Day CP, Anstee QM. Evidence of NAFLD progression from steatosis to fibrosingsteatohepatitis using paired biopsies: implications for prognosis and clinical management. J Hepatol 2015;62:1148-1155.

29. Villanova N, Moscatiello S, Ramilli S, et al. Endothelial dysfunction and cardiovascular risk profile in nonalcoholic fatty liver disease. Hepatology 2005;42:473-480.

30. Sung KC, Wild SH, Kwag HJ, Byrne CD. Fatty liver, insulin resistance, and features of metabolic syndrome: relationships with coronary artery calcium in 10,153 people. Diabetes Care 2012;35:2359-2364

31. Vanni E, Marengo A, Mezzabotta L, Bugianesi E. Systemic complications of nonalcoholic fatty liver disease: when the liver is not an innocent bystander. Semin Liver Dis 2015;35:236-249.

32. Arkan MC, Hevener AL, Greten FR, et al. IKK-beta links in- 
flammation to obesity-induced insulin resistance. Nat Med 2005;11:191-198.

33. Fabbrini E, Yoshino J, Yoshino M, et al. Metabolically normal obese people are protected from adverse effects following weight gain. J Clin Invest 2015;125:787-795.

34. Goland S, Shimoni S, Zornitzki T, et al. Cardiac abnormalities as a new manifestation of nonalcoholic fatty liver disease: echocardiographic and tissue Doppler imaging assessment. J Clin Gastroenterol 2006;40:949-955.

35. Chatrath H, Vuppalanchi R, Chalasani N. Dyslipidemia in patients with nonalcoholic fatty liver disease. Semin Liver Dis 2012;32:2229.

36. Hui JM, Hodge A, Farrell GC, Kench JG, Kriketos A, George J. Beyond insulin resistance in NASH: TNF-alpha or adiponectin? Hepatology 2004;40:46-54.

37. Petta S, Cammà C, Cabibi D, Di Marco V, Craxì A. Hyperuricemia is associated with histological liver damage in patients with non-alcoholic fatty liver disease. Aliment Pharmacol Ther 2011;34:757-766.

38. Sirota JC, McFann K, Targher G, Johnson RJ, Chonchol M, Jalal DI. Elevated serum uric acid levels are associated with nonalcoholic fatty liver disease independently of metabolic syndrome features in the United States: liver ultrasound data from the National Health and Nutrition Examination Survey. Metabolism 2013;62:392-399.

39. Targher G. High-sensitivity C-reactive protein, obesity, and subclinical atherosclerosis: implications of JUPITER from the MESA study. Arterioscler Thromb Vasc Biol 2011;31:1251-1252.

40. Targher G. Relationship between high-sensitivity C-reactive protein levels and liver histology in subjects with non-alcoholic fatty liver disease. J Hepatol 2006;45:879-881.

41. Softic S, Boucher J, Solheim MH, et al. Lipodystrophy due to adipose tissue-specific insulin receptor knockout results in progressive NAFLD. Diabetes 2016;65:2187-2200.

42. Bugianesi E, Pagotto U, Manini R, et al. Plasma adiponectin in nonalcoholic fatty liver is related to hepatic insulin resistance and hepatic fat content, not to liver disease severity. J Clin Endocrinol Metab 2005;90:3498-3504.

43. Kaser S, Moschen A, Cayon A, et al. Adiponectin and its receptors in non-alcoholic steatohepatitis. Gut 2005;54:117-121.

44. Targher G, Bertolini L, Rodella S, et al. Associations between plasma adiponectin concentrations and liver histology in patients with nonalcoholic fatty liver disease. Clin Endocrinol (Oxf) 2006;64:679-683.

45. Yamaji T, Iwasaki M, Sasazuki S, Tsugane S. Interaction between adiponectin and leptin influences the risk of colorectal adenoma. Cancer Res 2010;70:5430-5437.

46. Sookoian S, Castaño GO, Burgueño AL, et al. Circulating levels and hepatic expression of molecular mediators of atherosclerosis in nonalcoholic fatty liver disease. Atherosclerosis 2010;209:585591.

47. Targher G, Chonchol M, Miele L, Zoppini G, Pichiri I, Muggeo
M. Nonalcoholic fatty liver disease as a contributor to hypercoagulation and thrombophilia in the metabolic syndrome. Semin Thromb Hemost 2009;35:277-287.

48. Wieckowska A, Papouchado BG, Li Z, Lopez R, Zein NN, Feldstein AE. Increased hepatic and circulating interleukin-6 levels in human nonalcoholic steatohepatitis. Am J Gastroenterol 2008;103:1372-1379.

49. Tilg H, Moschen AR. Adipocytokines: mediators linking adipose tissue, inflammation and immunity. Nat Rev Immunol 2006;6:772-783.

50. Sookoian S, Gianotti TF, Rosselli MS, Burgueño AL, Castaño GO, Pirola CJ. Liver transcriptional profile of atherosclerosis-related genes in human nonalcoholic fatty liver disease. Atherosclerosis 2011;218:378-385.

51. Weston CJ, Shepherd EL, Claridge LC, et al. Vascular adhesion protein-1 promotes liver inflammation and drives hepatic fibrosis. J Clin Invest 2015;125:501-520.

52. Oni ET, Agatston AS, Blaha MJ, et al. A systematic review: burden and severity of subclinical cardiovascular disease among those with nonalcoholic fatty liver; should we care? Atherosclerosis 2013;230:258-267.

53. Kim HC, Kim DJ, Huh KB. Association between nonalcoholic fatty liver disease and carotid intima-media thickness according to the presence of metabolic syndrome. Atherosclerosis 2009;204:521525.

54. Brea A, Mosquera D, Martín E, Arizti A, Cordero JL, Ros E. Nonalcoholic fatty liver disease is associated with carotid atherosclerosis: a case-control study. Arterioscler Thromb Vasc Biol 2005;25:1045-1050

55. Fracanzani AL, Burdick L, Raselli S, et al. Carotid artery intimamedia thickness in nonalcoholic fatty liver disease. Am J Med 2008;121:72-78

56. Sookoian S, Pirola CJ. Non-alcoholic fatty liver disease is strongly associated with carotid atherosclerosis: a systematic review. J Hepatol 2008;49:600-607.

57. Chung GE, Choi SY, Kim D, et al. Nonalcoholic fatty liver disease as a risk factor of arterial stiffness measured by the cardioankle vascular index. Medicine (Baltimore) 2015;94:e654.

58. Salvi P, Ruffini R, Agnoletti D, et al. Increased arterial stiffness in nonalcoholic fatty liver disease: the Cardio-GOOSE study. J Hypertens 2010;28:1699-1707.

59. Lee YJ, Shim JY, Moon BS, et al. The relationship between arterial stiffness and nonalcoholic fatty liver disease. Dig Dis Sci 2012;57:196-203.

60. Pacifico L, Anania C, Martino F, et al. Functional and morphological vascular changes in pediatric nonalcoholic fatty liver disease. Hepatology 2010;52:1643-1651.

61. Kim D, Choi SY, Park EH, et al. Nonalcoholic fatty liver disease is associated with coronary artery calcification. Hepatology 2012;56:605-613.

62. Wong VW, Wong GL, Yip GW, et al. Coronary artery disease and cardiovascular outcomes in patients with non-alcoholic fatty liver 
disease. Gut 2011:60:1721-1727.

63. Sung KC, Kim SH. Interrelationship between fatty liver and insulin resistance in the development of type 2 diabetes. J Clin Endocrinol Metab 2011;96:1093-1097.

64. Wong VW, Chu WC, Wong GL, et al. Prevalence of non-alcoholic fatty liver disease and advanced fibrosis in Hong Kong Chinese: a population study using proton-magnetic resonance spectroscopy and transient elastography. Gut 2012;61:409-415.

65. Park HE, Kwak MS, Kim D, Kim MK, Cha MJ, Choi SY. Nonalcoholic fatty liver disease is associated with coronary artery calcification development: a longitudinal study. J Clin Endocrinol Metab 2016;101:3134-3143.

66. Choi SY, Kim D, Kim HJ, et al. The relation between non-alcoholic fatty liver disease and the risk of coronary heart disease in Koreans. Am J Gastroenterol 2009;104:1953-1960.

67. Targher G, Byrne CD, Lonardo A, Zoppini G, Barbui C. Nonalcoholic fatty liver disease and risk of incident cardiovascular disease: a meta-analysis. J Hepatol 2016;65:589-600.

68. Ekstedt M, Hagström H, Nasr P, et al. Fibrosis stage is the strongest predictor for disease-specific mortality in NAFLD after up to 33 years of follow-up. Hepatology 2015;61:1547-1554.

69. Vilar-Gomez E, Calzadilla-Bertot L, Wai-Sun Wong V, et al. Fibrosis severity as a determinant of cause-specific mortality in patients with advanced nonalcoholic fatty liver disease: a multinational cohort study. Gastroenterology 2018;155:443-457.

70. Ahn AL, Choi JK, Kim MN, et al. Non-alcoholic fatty liver disease and chronic kidney disease in Koreans aged 50 years or older. Korean J Fam Med 2013;34:199-205.

71. Jia G, Di F, Wang Q, et al. Non-alcoholic fatty liver disease is a risk factor for the development of diabetic nephropathy in patients with type 2 diabetes mellitus. PLoS One 2015;10:e0142808.

72. Mikolasevic I, Racki S, Bubic I, Jelic I, Stimac D, Orlic L. Chronic kidney disease and nonalcoholic Fatty liver disease proven by transient elastography. Kidney Blood Press Res 2013;37:305-310.

73. Pacifico L, Bonci E, Andreoli GM, et al. The impact of nonalcoholic fatty liver disease on renal function in children with overweight/obesity. Int J Mol Sci 2016;17:E1218.

74. Pan LL, Zhang HJ, Huang ZF, et al. Intrahepatic triglyceride content is independently associated with chronic kidney disease in obese adults: a cross-sectional study. Metabolism 2015;64:10771085.

75. Sirota JC, McFann K, Targher G, Chonchol M, Jalal DI. Association between nonalcoholic liver disease and chronic kidney disease: an ultrasound analysis from NHANES 1988-1994. Am J Nephrol 2012;36:466-471.

76. Xu HW, Hsu YC, Chang CH, Wei KL, Lin CL. High FIB-4 index as an independent risk factor of prevalent chronic kidney disease in patients with nonalcoholic fatty liver disease. Hepatol Int 2016;10:340-346

77. Yilmaz Y, Alahdab YO, Yonal 0, et al. Microalbuminuria in nondiabetic patients with nonalcoholic fatty liver disease: association with liver fibrosis. Metabolism 2010;59:1327-1330.
78. Yasui K, Sumida Y, Mori Y, et al. Nonalcoholic steatohepatitis and increased risk of chronic kidney disease. Metabolism 2011;60:735-739.

79. Machado MV, Gonçalves S, Carepa F, Coutinho J, Costa A, Cortez-Pinto H. Impaired renal function in morbid obese patients with nonalcoholic fatty liver disease. Liver Int 2012;32:241-248.

80. Musso G, Gambino R, Tabibian JH, et al. Association of nonalcoholic fatty liver disease with chronic kidney disease: a systematic review and meta-analysis. PLoS Med 2014;11:e1001680.

81. Vilar-Gomez E, Calzadilla-Bertot L, Friedman SL, et al. Improvement in liver histology due to lifestyle modification is independently associated with improved kidney function in patients with non-alcoholic steatohepatitis. Aliment Pharmacol Ther 2017;45:332-344.

82. Musso G, Cassader M, Cohney S, et al. Fatty liver and chronic kidney disease: novel mechanistic insights and therapeutic opportunities. Diabetes Care 2016;39:1830-1845.

83. Chung GE, Kim D, Kim W, et al. Non-alcoholic fatty liver disease across the spectrum of hypothyroidism. J Hepatol 2012;57:150156.

84. Pagadala MR, Zein CO, Dasarathy S, Yerian LM, Lopez R, McCullough AJ. Prevalence of hypothyroidism in nonalcoholic fatty liver disease. Dig Dis Sci 2012;57:528-534.

85. Kim D, Kim W, Joo SK, Bae JM, Kim JH, Ahmed A. Subclinical hypothyroidism and low-normal thyroid function are associated with nonalcoholic steatohepatitis and fibrosis. Clin Gastroenterol Hepatol 2018;16:123-131.

86. Kim D, Yoo ER, Li AA, et al. Low-normal thyroid function is associated with advanced fibrosis among adults in the United States. Clin Gastroenterol Hepatol 2019;17:2379-2381.

87. Manka PP, Coombes JD, Bechmann LP, et al. Thyroid hormone receptor regulates hepatic stellate cell activation. J Hepatol 2017;66(1 Suppl):S582.

88. Kelly MJ, Pietranico-Cole S, Larigan JD, et al. Discovery of 2-[3,5-dichloro-4-(5-isopropyl-6-oxo-1,6-dihydropyridazin3-yloxy)phenyl]-3,5-dioxo-2,3,4,5-tetrahydro[1,2,4]triazine6-carbonitrile (MGL-3196), a highly selective thyroid hormone receptor $\beta$ agonist in clinical trials for the treatment of dyslipidemia. J Med Chem 2014;57:3912-3923.

89. Kowalik MA, Columbano A, Perra A. Thyroid hormones, thyromimetics and their metabolites in the treatment of liver disease. Front Endocrinol (Lausanne) 2018;9:382.

90. March WA, Moore VM, Willson KJ, Phillips DI, Norman RJ, Davies MJ. The prevalence of polycystic ovary syndrome in a community sample assessed under contrasting diagnostic criteria. Hum Reprod 2010;25:544-551.

91. Kelley CE, Brown AJ, Diehl AM, Setji TL. Review of nonalcoholic fatty liver disease in women with polycystic ovary syndrome. World J Gastroenterol 2014;20:14172-14184.

92. Brzozowska MM, Ostapowicz G, Weltman MD. An association between non-alcoholic fatty liver disease and polycystic ovarian syndrome. J Gastroenterol Hepatol 2009;24:243-247. 
93. Ramezani-Binabaj M, Motalebi M, Karimi-Sari H, Rezaee-Zavareh MS, Alavian SM. Are women with polycystic ovarian syndrome at a high risk of non-alcoholic Fatty liver disease; a metaanalysis. Hepat Mon 2014;14:e23235.

94. Rocha ALL, Faria LC, Guimarães TCM, et al. Non-alcoholic fatty liver disease in women with polycystic ovary syndrome: systematic review and meta-analysis. J Endocrinol Invest 2017;40:12791288.

95. Wu J, Yao XY, Shi RX, Liu SF, Wang XY. A potential link between polycystic ovary syndrome and non-alcoholic fatty liver disease: an update meta-analysis. Reprod Health 2018;15:77.

96. Legro RS, Castracane VD, Kauffman RP. Detecting insulin resistance in polycystic ovary syndrome: purposes and pitfalls. Obstet Gynecol Surv 2004;59:141-154.

97. Karoli R, Fatima J, Chandra A, Gupta U, Islam FU, Singh G. Prevalence of hepatic steatosis in women with polycystic ovary syndrome. J Hum Reprod Sci 2013;6:9-14.

98. Gambarin-Gelwan M, Kinkhabwala SV, Schiano TD, Bodian C, Yeh HC, Futterweit W. Prevalence of nonalcoholic fatty liver disease in women with polycystic ovary syndrome. Clin Gastroenterol Hepatol 2007;5:496-501.

99. Targher G, Solagna E, Tosi F, et al. Abnormal serum alanine aminotransferase levels are associated with impaired insulin sensitivity in young women with polycystic ovary syndrome. J Endocrinol Invest 2009;32:695-700.

100. Kim JJ, Kim D, Yim JY, et al. Polycystic ovary syndrome with hyperandrogenism as a risk factor for non-obese non-alcoholic fatty liver disease. Aliment Pharmacol Ther 2017;45:1403-1412.

101. Ganzetti G, Campanati A, Offidani A. Non-alcoholic fatty liver disease and psoriasis: so far, so near. World J Hepatol 2015;7:315-326.

102. Gisondi P, Fostini AC, Fossà I, Girolomoni G, Targher G. Psoriasis and the metabolic syndrome. Clin Dermatol 2018;36:21-28.

103. Mallbris L, Ritchlin CT, Ståhle M. Metabolic disorders in patients with psoriasis and psoriatic arthritis. Curr Rheumatol Rep 2006;8:355-363.

104. Roberts KK, Cochet AE, Lamb PB, et al. The prevalence of NAFLD and NASH among patients with psoriasis in a tertiary care dermatology and rheumatology clinic. Aliment Pharmacol Ther 2015;41:293-300.

105. van der Voort EA, Koehler EM, Dowlatshahi EA, et al. Psoriasis is independently associated with nonalcoholic fatty liver disease in patients 55 years old or older: results from a population-based study. J Am Acad Dermatol 2014;70:517-524.

106. Gisondi P, Targher G, Zoppini G, Girolomoni G. Non-alcoholic fatty liver disease in patients with chronic plaque psoriasis. $\mathrm{J}$ Hepatol 2009;51:758-764.

107. Miele L, Vallone S, Cefalo C, et al. Prevalence, characteristics and severity of non-alcoholic fatty liver disease in patients with chronic plaque psoriasis. J Hepatol 2009;51:778-786.

108. Campanati A, Ganzetti G, Di Sario A, et al. The effect of etanercept on hepatic fibrosis risk in patients with non-alcoholic fatty liver disease, metabolic syndrome, and psoriasis. J Gastroenterol 2013;48:839-846.

109. Prussick RB, Miele L. Nonalcoholic fatty liver disease in patients with psoriasis: a consequence of systemic inflammatory burden? Br J Dermatol 2018;179:16-29.

110. Arnett DK, Blumenthal RS, Albert MA, et al. 2019 ACC/AHA guideline on the primary prevention of cardiovascular disease. Circulation 2019;140:e596-e646.

111. Kirwan JP, Sacks J, Nieuwoudt S. The essential role of exercise in the management of type 2 diabetes. Cleve Clin J Med 2017;84(7 Suppl 1):S15-S21.

112. VanWagner LB, Rinella ME. Extrahepatic manifestations of nonalcoholic fatty liver disease. Curr Hepatol Rep 2016;15:75-85.

113. Chalasani N, Younossi Z, Lavine JE, et al. The diagnosis and management of non-alcoholic fatty liver disease: practice guideline by the American Gastroenterological Association, American Association for the Study of Liver Diseases, and American College of Gastroenterology. Gastroenterology 2012;142:1592-1609.

114. Paulweber B, Valensi P, Lindström J, et al. A European evidencebased guideline for the prevention of type 2 diabetes. Horm Metab Res 2010;42 Suppl 1:S3-36.

115. Golay A, Brock E, Gabriel R, et al. Taking small steps towards targets: perspectives for clinical practice in diabetes, cardiometabolic disorders and beyond. Int J Clin Pract 2013;67:322-332.

116. Shen J, Wong GL, Chan HL, et al. PNPLA3 gene polymorphism and response to lifestyle modification in patients with nonalcoholic fatty liver disease. J Gastroenterol Hepatol 2015;30:139146.

117. Sevastianova K, Kotronen A, Gastaldelli A, et al. Genetic variation in PNPLA3 (adiponutrin) confers sensitivity to weight loss-induced decrease in liver fat in humans. Am J Clin Nutr 2011;94:104-111.

118. Marzuillo P, Grandone A, Perrone L, del Giudice EM. Weight loss allows the dissection of the interaction between abdominal fat and PNPLA3 (adiponutrin) in the liver damage of obese children. J Hepatol 2013;59:1143-1144.

119. Jensen MD, Ryan DH, Apovian CM, et al. 2013 AHA/ACC/TOS guideline for the management of overweight and obesity in adults: a report of the American College of Cardiology/American Heart Association Task Force on Practice Guidelines and The Obesity Society. Circulation 2014;129(25 Suppl 2):S102-S138.

120. Zelber-Sagi S, Nitzan-Kaluski D, Goldsmith R, et al. Long term nutritional intake and the risk for non-alcoholic fatty liver disease (NAFLD): a population based study. J Hepatol 2007;47:711717.

121. Ferolla SM, Silva LC, Ferrari Mde L, et al. Dietary approach in the treatment of nonalcoholic fatty liver disease. World J Hepatol 2015;7:2522-2534.

122. Assy N, Nasser G, Kamayse I, et al. Soft drink consumption linked with fatty liver in the absence of traditional risk factors. Can J Gastroenterol 2008;22:811-816.

123. van der Poorten D, Milner KL, Hui J, et al. Visceral fat: a key 
mediator of steatohepatitis in metabolic liver disease. Hepatology 2008;48:449-457.

124. Zelber-Sagi S, Nitzan-Kaluski D, Goldsmith R, et al. Role of leisure-time physical activity in nonalcoholic fatty liver disease: a population-based study. Hepatology 2008;48:1791-1798.

125. Magkos F. Exercise and fat accumulation in the human liver. Curr Opin Lipidol 2010;21:507-517.

126. Hallsworth K, Fattakhova G, Hollingsworth KG, et al. Resistance exercise reduces liver fat and its mediators in non-alcoholic fatty liver disease independent of weight loss. Gut 2011;60:1278-1283.

127. Bae JC, Suh S, Park SE, et al. Regular exercise is associated with a reduction in the risk of NAFLD and decreased liver enzymes in individuals with NAFLD independent of obesity in Korean adults. PLoS One 2012;7:e46819.

128. Tamura Y, Tanaka Y, Sato F, et al. Effects of diet and exercise on muscle and liver intracellular lipid contents and insulin sensitivity in type 2 diabetic patients. J Clin Endocrinol Metab 2005;90:3191-3196.

129. Kwak MS, Kim D, Chung GE, Kim W, Kim YJ, Yoon JH. Role of physical activity in nonalcoholic fatty liver disease in terms of visceral obesity and insulin resistance. Liver Int 2015;35:944-952.

130. Kwak MS, Kim D, Chung GE, Kim W, Kim JS. The preventive effect of sustained physical activity on incident nonalcoholic fatty liver disease. Liver Int 2017;37:919-926. 